\title{
Second-harmonic generation in silicon waveguides strained by silicon nitride
}

\author{
M. Cazzanelli', F. Bianco', E. Borga' ', G. Pucker ${ }^{2}$, M. Ghulinyan² ${ }^{2}$, E. Degoli ${ }^{3}$, E. Luppi ${ }^{4}$, V. Véniard ${ }^{5}$, \\ S. Ossicini ${ }^{3}$, D. Modotto ${ }^{6}$, S. Wabnitz ${ }^{6}$, R. Pierobon ${ }^{7}$ and L. Pavesi ${ }^{1 \star}$
}

Silicon photonics meets the electronics requirement of increased speed and bandwidth with on-chip optical networks. All-optical data management requires nonlinear silicon photonics. In silicon only third-order optical nonlinearities are present owing to its crystalline inversion symmetry. Introducing a second-order nonlinearity into silicon photonics by proper material engineering would be highly desirable. It would enable devices for wideband wavelength conversion operating at relatively low optical powers. Here we show that a sizeable second-order nonlinearity at optical wavelengths is induced in a silicon waveguide by using a stressing silicon nitride overlayer. We carried out second-harmonic-generation experiments and first-principle calculations, which both yield large values of strain-induced bulk second-order nonlinear susceptibility, up to 40 pm $\mathbf{V}^{-1}$ at $2,300 \mathrm{~nm}$. We envisage that nonlinear strained silicon could provide a competing platform for a new class of integrated light sources spanning the near- to mid-infrared spectrum from 1.2 to $10 \mu \mathrm{m}$.

W hen a crystal possesses a significant second-order nonlinear optical susceptibility, $\chi^{(2)}$, it can produce a wide variety of wavelengths from an optical pump ${ }^{1}$. In fact, a second-order crystal generates shorter wavelengths by second-harmonic generation or longer wavelengths by spontaneous parametric down-conversion of a single pump beam. Such a crystal can also nonlinearly mix two different beams, thus generating other wavelengths by sum-frequency or difference-frequency generation. These possibilities are much more intriguing whenever the crystal can be used in integrated optical circuits because, on the one hand, light confinement reduces the average optical power needed to trigger nonlinear processes and, on the other hand, relatively long effective interaction lengths can be exploited.

$\mathrm{Si}$ photonics has demonstrated the integration of multiple optical functionalities with microelectronic devices ${ }^{2,3}$. On the basis of the third- or higher-order nonlinearities of Si (ref. 4), functions such as amplification and lasing, wavelength conversion and optical processing have all been demonstrated in recent years ${ }^{5}$. However, third-order refractive nonlinearities require relatively high optical powers, and compete with nonlinear-loss mechanisms such as two-photon absorption and two-photon induced freecarrier absorption. Yet, the second-order term of the nonlinear susceptibility tensor cannot be exploited in Si simply because $\chi^{(2)}$ vanishes in the dipole approximation owing to the crystal centrosymmetry: the residual $\chi^{(2)}$, which is due to higher-multipole processes, is too weak to be exploited in optical devices ${ }^{6}$.

Second-harmonic generation (SHG) was observed in reflection from $\mathrm{Si}$ surfaces $^{7-11}$ or in diffusion from $\mathrm{Si}$ photonic crystal nanocavities ${ }^{12}$. This indicates that the reduction of the Si symmetry may indeed induce a significant $\chi^{(2)}$. In these cases, the Si symmetry was broken by the presence of a surface. Several groups have pointed out that the surface contribution to $\chi^{(2)}$ can be strengthened by applying a strain ${ }^{8-10}$. It was also reported that strain and increased internal surfaces in a Si photonic crystal waveguide may induce a strong $\chi^{(2)}$, which enables measurement of electro-optic effects ${ }^{13,14}$. Theoretically, these results are so far qualitatively but not quantitatively understood ${ }^{15}$. The prospect of controlling second-order nonlinear phenomena in Si by means of strain is very intriguing. In this work we provide the first quantitative demonstration of second-order nonlinearities in strained Si through both ab initio calculations and SHG experiments in suitably engineered $\mathrm{Si}$ waveguides.

Second-order nonlinear optical response from strained bulk $\mathrm{Si}$ is computed by time-dependent density-functional theory in a supercell approximation with periodic boundary conditions ${ }^{16-18}$. All the studied strained Si structures were obtained using a unit cell of $16 \mathrm{Si}$ atoms (Fig. 1) initially in their bulk centro-symmetric positions (see Supplementary Information). We broke the Si symmetry by moving the Si atoms in the lattice, that is by modifying the Si bond lengths and angles. This yields a strained Si model which enables us to calculate the second-order response in Si. When no strain or a uniformly distributed strain is simulated, the computed $\chi^{(2)}$ vanishes because bulk $\mathrm{Si}$ still keeps a crystalline centrosymmetry. To break the symmetry, the stress has to induce an asymmetric deformation of the Si lattice. We model this situation by moving only sets of atoms in the supercell (Fig. 1a) simulating tensile $\left(\mathbf{S}_{\mathrm{t}}\right)$ and/or compressive $\left(\mathbf{S}_{\mathrm{c}}\right)$ stresses. With this approach we identify and study two classes of systems: $\mathbf{U S}_{\mathrm{c}} \mathbf{U S}_{\mathrm{t}}$ (unstrained-compressed-unstrained-tensile) and $\mathrm{US}_{\mathrm{t}} \mathbf{U S}_{\mathrm{t}}$ (unstrained-tensile-unstrained-tensile; see Supplementary Information). Simulation results show that $\chi^{(2)} \neq 0$ whenever the unit cell is inhomogeneously strained. We also observed that $\chi^{(2)}$ is related to the local lattice deformation (that is, the local strain): the more the lattice is distorted the larger is the $\chi^{(2)}$ value. Moving the atoms in $\mathbf{S}_{\mathrm{t}} / \mathbf{S}_{\mathrm{c}}$ regions along the (001) direction only, we found that

\footnotetext{
${ }^{1}$ Nanoscience Laboratory, Department of Physics, University of Trento, via Sommarive 14, 38123 Povo, Trento, Italy, ${ }^{2}$ Advanced Photonics \& Photovoltaics Unit, Bruno Kessler Foundation, via Sommarive 18, 38123 Povo, Trento, Italy, ${ }^{3}$ Istituto di Nanoscienze-CNR-S3 and Dipartimento di Scienze e Metodi dell'Ingegneria, Università di Modena e Reggio Emilia, via Amendola 2 Pad. Morselli, I-42122 Reggio Emilia, Italy, ${ }^{4}$ Department of Chemistry, University of California Berkeley, California 94720, USA, ${ }^{5}$ Laboratoire des Solides Irradiés, Ecole Polytechnique, Route de Saclay, F-91128 Palaiseau and European Theoretical Spectroscopy Facility (ETSF), France, ${ }^{6}$ Department of Information Engineering, University of Brescia, via Branze 38, 25123 Brescia, Italy, ${ }^{7}$ CIVEN, via delle Industrie 5, I-30175, Venezia Marghera, Italy. *e-mail: pavesi@science.unitn.it.
} 

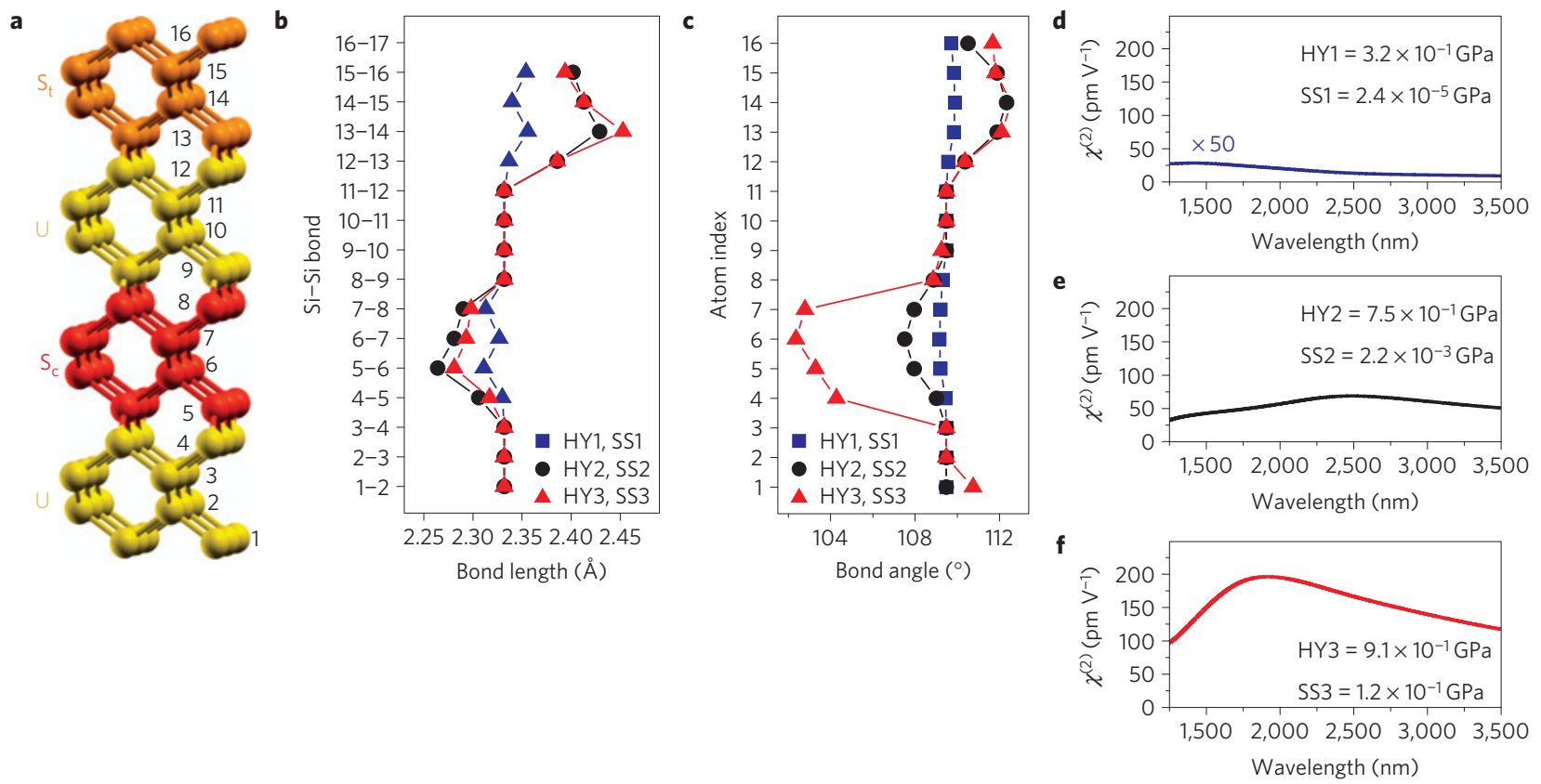

Figure 1 | First-principle calculations of strained Si second-order nonlinearity. a, Simulation unit cell: the different colours refer to the different bond deformations used. Orange: the bonds were elongated to represent a tensile stress, indicated by $\mathbf{S}_{\mathrm{t}}$. Yellow: no bond variation with respect to the relaxed $\mathrm{Si}$ lattice (unperturbed region). Red: the bonds were shortened to represent a compressive stress, indicated by $\mathbf{S}_{\mathrm{c}}$. $\mathbf{b}$, Maximum bond-length distortions for $\mathbf{U S}_{\mathrm{c}} \mathbf{U} \mathbf{S}_{\mathrm{t}}$ structures. The unstrained bulk-Si bond length is $2.332 \AA$. The colours (blue-black-red) refer to the calculations shown in $\mathbf{d}-\mathbf{f}$. c $\mathbf{c}$, Maximum bond-angle distortions for $\mathbf{U S}_{\mathrm{C}} \mathbf{U S}_{\mathrm{t}}$ structures. The unstrained bulk-Si angles are 109.5. The colours (blue-black-red) refer to the calculations shown in $\mathbf{d}-\mathbf{f}$. The constant bond lengths and angles represent the unstrained region and coincide with Si-bulk values. The hierarchy of increasing strain in the three structures (blue-black-red), which is reflected also in the corresponding values of pressure (HY) and in the YZ component of the shear stress (SS; see Supplementary Information), is evident. $\mathbf{d}-\mathbf{f}$, The results of the simulations in terms of the $\chi^{(2)}$ values. $\mathbf{d}, \mathrm{A}$ weakly strained Si. e, A medium-strained Si. f, A heavily strained Si.

$\chi^{(2)}$ is lower than $0.5 \mathrm{pm} \mathrm{V}^{-1}$ at $2,500 \mathrm{~nm}$. In contrast, if we increase the strain inhomogeneity moving the atoms also in the (110) direction, we found that $\chi^{(2)}$ increases significantly (Fig. 1). For each class, $\mathbf{U S}_{\mathrm{c}} \mathbf{U S}_{\mathrm{t}}$ and $\mathbf{U S}_{\mathrm{t}} \mathbf{U S} \mathbf{S}_{\mathrm{t}}$, we studied three structures, in which we have progressively increased the applied strain. In Fig. 1b,c we show the maximum structural distortion in term of bond lengths and angles for the $\mathbf{U S}_{\mathrm{c}} \mathbf{U S} \mathbf{S}_{\mathrm{t}}$ structures (the same can be found for the $\mathbf{U S}_{\mathrm{t}} \mathbf{U S}_{\mathrm{t}}$ structures; see Supplementary Information). The type and magnitude of strain is qualitatively observed as bond-length and angle deviations with respect to the unstrained Si bulk values. We observe that $\chi^{(2)}$ has a maximum of $6 \mathrm{pm} \mathrm{V}^{-1}$ at a wavelength of $1,900 \mathrm{~nm}$ for purely tensile strain. The magnitude of the applied strain was up to $5 \%$ bond distortion of the unstrained bulk value, that is up to $0.12 \AA$. When compressive and tensile strains are applied (Fig. 1), $\chi^{(2)}$ increases further, reaching a maximum value of about $200 \mathrm{pm} \mathrm{V}^{-1}$ at $1,800 \mathrm{~nm}$, as shown in Fig. 1f. In this case, the strain gradient is larger than that of the pure-tensile-strain case, because of the change in sign of the strain itself between $S_{t}$ and $S_{c}$ (Fig. 1b,c). As the reported calculations refer to a strained bulk $\mathrm{Si}$, we also investigate the contribution to $\chi^{(2)}$ from a $\mathrm{Si} / \mathrm{SiO}_{2}$ interface. We found a $\chi^{(2)}$ smaller than $1 \mathrm{pm} \mathrm{V}^{-1}$ (Supplementary Information), whereas from the literature it is found that the free $\mathrm{Si}$ surface induces a $\chi^{(2)}$ of $\sim 3 \mathrm{pm} \mathrm{V}^{-1}$ (refs 11,19). Therefore, breaking the $\mathrm{Si}$ inversion symmetry by an interface or a free surface yields a $\chi^{(2)}$ that is significantly smaller than what can be achieved by inhomogeneously straining bulk $\mathrm{Si}$.

To explore this concept, we fabricated inhomogeneously strained Si waveguides. These were obtained by using Si-oninsulator (SOI) waveguides (Fig. 2) with stressing $\mathrm{SiN}_{x}$ overlayer clads (Methods section). Various cladding layers were used to change the applied stress: the waveguide named SOI1 had a 150-nm-thick $\mathrm{Si}_{3} \mathrm{~N}_{4}$ overlayer, which induces a tensile stress of
1.2 GPa; the waveguide named SOI2 had a 500-nm-thick $\mathrm{SiN}_{x}$ overlayer, which induces a compressive stress of $-500 \mathrm{MPa}$; the waveguide named SOI3 had a stress-compensated 500-nm-thick $\mathrm{SiN}_{x}$ overlayer with a residual compressive stress of $-60 \mathrm{MPa}$. Note that the reported stress values refer to measurements made on the whole unpatterned Si wafers by using a mechanical profilometer. Next, waveguides were defined by optical lithography and by reactive ion etching (Fig. 2c). The light propagation axis, along the waveguide, was parallel to the (110) crystalline direction (Fig. 2b). Our multimode waveguides exhibited linear propagation losses of $1-3 \mathrm{~dB} \mathrm{~cm}^{-1}$ at $1,260 \mathrm{~nm}$. Control waveguides without the stressing overlayer were also fabricated (named SOI0).

The silicon nitride overlayer strains the $\mathrm{Si}$ waveguide because of the stress penetration into the waveguide core. To map the strain distribution in the waveguide cross-section we used spatially resolved (with a $0.5 \mu \mathrm{m}$ resolution) micro-Raman spectroscopy. The strain maps were constructed by acquiring the Raman spectrum on each point of the waveguide cross-section. The shift in the Si phonon frequency with respect to the unperturbed phonon frequency, which was measured on the substrate, can be related to the local $\mathrm{Si}$ strain by a simple uniaxial model ${ }^{20}$. Figure 3 shows the distribution of the strain components for the three sets of waveguides with $\mathrm{SiN}_{x}$ claddings. The tensile SOI1 sample (Fig. 3a,d,g,h) shows a significant deformation all along the waveguide core. Note that this means that the application of the stressing overlayer strains the whole $2-\mu \mathrm{m}$-thick waveguide (Fig. 3h). It can also be noticed that the underlying buried oxide (BOX) affects the strain distribution too. As a result, a very inhomogeneous strain field is observed: the applied stress results in a compressive strain in a thin layer below the $\mathrm{Si}_{3} \mathrm{~N}_{4}$ cladding layer and a tensile strain in the waveguide core, with a maximum value near the BOX interface. The strain field has a strong gradient, which, 


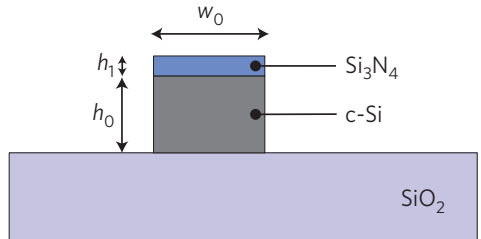

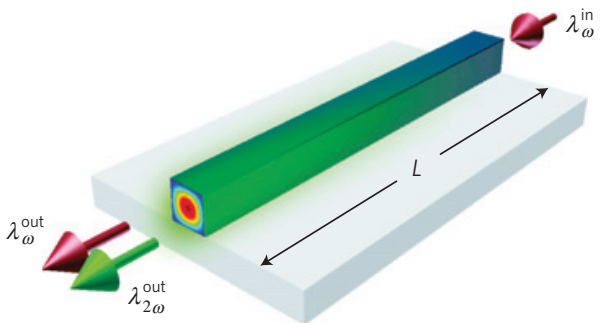
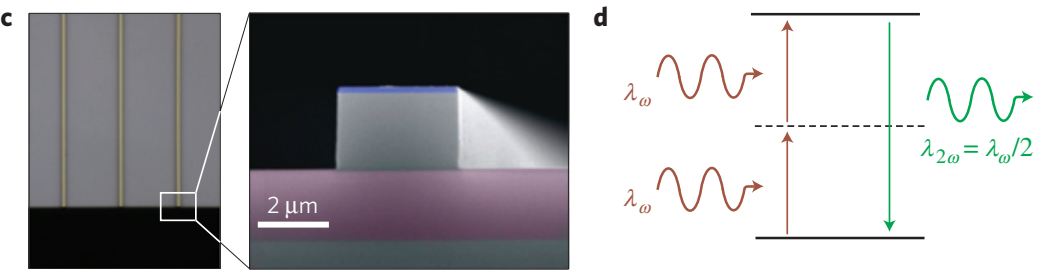

Figure 2 | Strained Si waveguides used to measure SHG. a, Schematic representation of the cross-section of a strained SOI waveguide where $w_{0}$ and $h_{0}$ are the width and height of the waveguide and $h_{1}$ the nitride overlayer's height. Si forms the core of the waveguide and the parameters are chosen to have more than $95 \%$ of the optical field confined within the waveguide. $\mathbf{b}$. The second-harmonic experiment, where $L$ is the total waveguide length. A pump pulse is coupled into the waveguide at a wavelength $\lambda_{\omega}$ and two pulses (at wavelengths $\lambda_{\omega}$ and $\lambda_{2 \omega}$ ) are measured at the output of the waveguide. c, $A$ top-view optical image of the sample where a few waveguides are observed as yellow lines. A scanning electron microscopy image of the input facet of the waveguide is also shown. The layers are false coloured as in $\mathbf{a}$. Note that the stressing overlayer does not produce macroscopic cracks or deformation in the strained Si waveguide. d, Virtual-energy-level diagram of SHG, where two photons of the pump at wavelength $\lambda_{\omega}$ are annihilated by way of a virtual energy level to create a single photon at wavelength $\lambda_{2 \omega}$

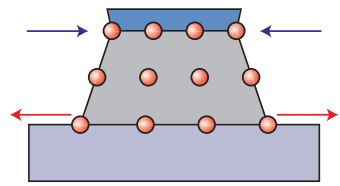

d

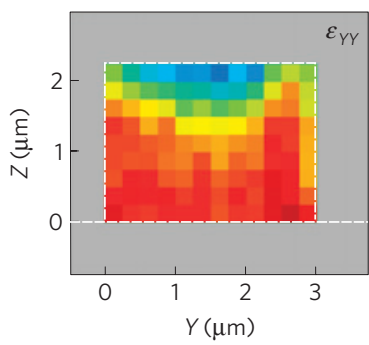

g

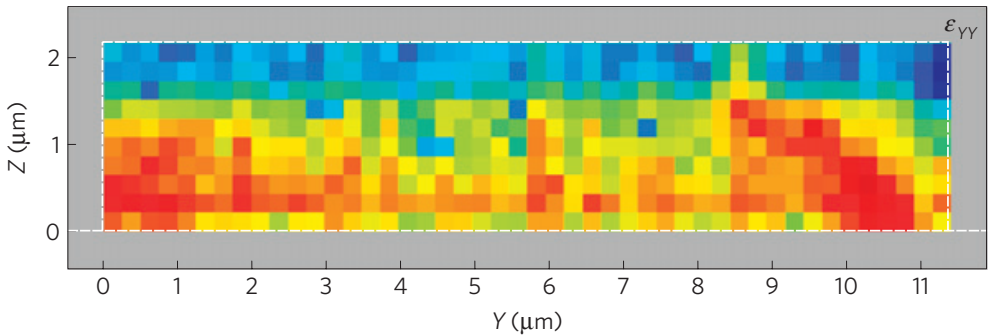

b
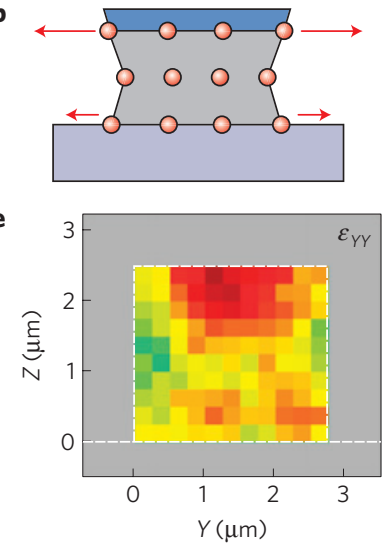

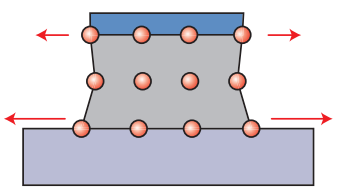

$\mathbf{f}$

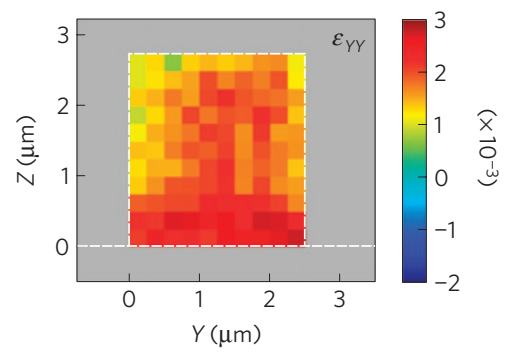

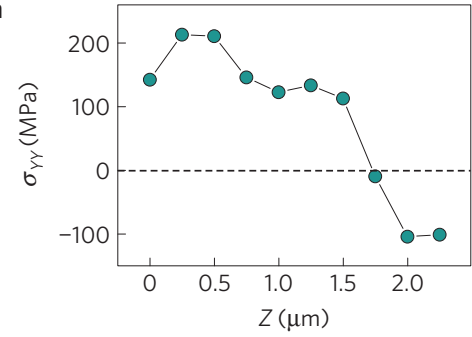

Figure 3 | Summary of the results of micro-Raman measurements on the waveguide facet. a-c, Schematic representations of the SOI1, SOI2 and SOI3 waveguides. The lattice deformation is exaggerated to show the effect of the applied stressing overlayer (blue layer). The arrows show the kind of tensile/compressive strain observed. d, Two-dimensional map of the measured strain-tensor element $\varepsilon_{Y Y}$ for the SOI1 2.3- $\mu \mathrm{m}$-wide waveguide. The colour bar is reported on the right. On the basis of this map the sketch in a was deduced. e, Two-dimensional map of the strain-tensor element $\varepsilon_{Y Y}$ for the SOI2 2.0- $\mu \mathrm{m}$-wide waveguide. $\mathbf{f}$, Two-dimensional map of the strain-tensor element $\varepsilon_{Y Y}$ for the SOI3 2.0- $\mu$ m-wide waveguide. $\mathbf{g}$, Two-dimensional map of the strain-tensor element $\varepsilon_{Y Y}$ for the SOI1 10.7- $\mu$ m-wide waveguide. $\mathbf{h}$, Line scan of the measured stress at the centre of the SOI1 10.7- $\mu$ m-wide waveguide. Note that for SOI1 the overlayer nitride film is tensile strained, which causes a compressive stress on a Si wafer, which when the waveguide is defined yields a compressive stress at the nitride/Si interface of the waveguide. This stress finally ends up with a sign change across the waveguide cross-section due to the presence of the underlying BOX layer.

according to calculations, should induce a significant $\chi^{(2)}$. The strain field has a non-zero component along the $Y$ axis (Fig. 3d), that is along the facet plane as sketched in Fig. 3a, a complementary weaker compressive component along the $Z$ axis, that is along the waveguide thickness, and a negligible component along the $X$ axis, that is along the waveguide axis. The strain along the $Y$ axis 


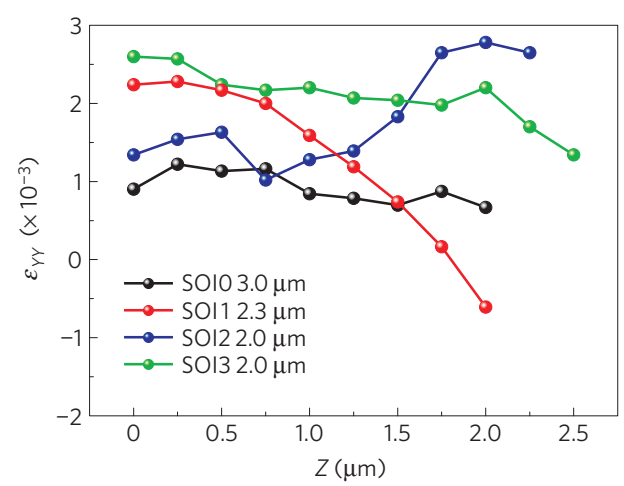

Figure 4 | Strain profiles ( $\varepsilon_{Y Y}$ ) for the different waveguide types. Profiles refer to the strain along the $Z$ direction at the centre of the waveguides. The waveguide widths are reported in the key.

also depends on the waveguide width: the wider the waveguide the more inhomogeneous the strain (compare Fig. $3 \mathrm{~d}$ with $3 \mathrm{~g}$ ). The situation is different for the compressed SOI2 waveguide (Fig. 3b,e): although here the $\mathrm{Si}$ is mainly tensile strained, an inhomogeneous strain field is still present. This field has a maximum near the overlayer and gradually weakens near the underlying BOX. SOI3 shows a strain field that is the opposite of SOI2 (Fig. $3 \mathrm{c}, \mathrm{f}$ ) due to the stronger effect of the BOX with respect to the overlayer. In Fig. 4 we report the $Z$-profile of the strain at the waveguide centre, for the various samples. SOI1 and SOI2 show important strain gradients of opposite signs, reflecting the influence of strong tensile and compressive stress applied by the $\mathrm{SiN}_{x}$ overlayers. Strain and its gradient are low for the reference SOI0 sample. SOI3 shows a similar strain gradient, but the average strain is about twice that of $\mathrm{SOI} 0$. In both samples the origin of strain is mainly due to the stress induced by the BOX.

To demonstrate the induced second-order nonlinearity we carried out SHG experiments (Fig. 2b,d). Two different pump lasers were used with 4-ns- and 100-fs-long pulses, respectively. Taking into account the temporal walk-off between the pump and the second-harmonic signal, 2-mm-long waveguides were used in combination with the fs laser. One-centimetre-long waveguides were used in combination with the ns laser, because in this case the pump-signal walk-off can be ignored. Figure 5 a shows a typical transmission signal recorded from the SOI1 $10.7-\mu \mathrm{m}$-wide waveguide for a $30-\mathrm{kW}$-peak-power fs pump. In addition to the residual pump spectrum centred at about $2,100 \mathrm{~nm}$, a significant second-harmonic peak at 1,050 nm was clearly observed (Fig. 5a). Note that the wavelength of the emitted radiation is half the pump wavelength, as expected for SHG. The second-harmonic peak shifts linearly with the pump wavelength (Fig. 5b) over a $300 \mathrm{~nm}$ range of variation of the pump wavelength. A further proof of the fact that the short-wavelength peak is due to SHG is provided by the observation of a characteristic quadratic dependence of the measured peak power as a function of ns pump peak power (Fig. 5c). The deviation from a quadratic dependence at high pump peak powers that is observed in Fig. $5 c$ is due to the pump depletion, which is caused by nonlinear optical losses 5 . Tentatively, these nonlinear losses can be attributed to three-photon absorption processes and/or to the possible two-photon absorption processes due to strain-induced bandgap shrinkage. Fig. 5d reports the variation of the second-harmonic peak power as a function of the ns pump wavelength for the SOI1 2.3- $\mu$ m-wide waveguide. No similar features in this pump power range were observed on control measurements on SOI0 waveguides, that is, those without the stressing overlayer.

At a wavelength of $2,313 \mathrm{~nm}$ and at a ns pump peak power of $0.7 \pm 0.1 \mathrm{~W}$, the power-conversion efficiency $\eta$ from the pump laser (peak power $P_{\omega}$ ) to the second-harmonic beam (peak power $\left.P_{2 \omega}\right)$ is $\eta=P_{2 \omega} / P_{\omega}=(5 \pm 3) \times 10^{-8} \mathrm{~W} \mathrm{~W}^{-1}=-73 \mathrm{~dB}\left(\right.$ or $P_{2 \omega} / P_{\omega}^{2}=$ $\left.(7.5 \pm 4) \times 10^{-8} \mathrm{~W}^{-1}\right)$ for the SOI1 $10.7-\mu \mathrm{m}$-wide waveguide. Table 1 reports the $\eta$ values for the various waveguides and stressing overlayers. In agreement with the Raman measurements, which show that the SOI1 waveguides are more strained than the other sets of waveguides, the tensile-stressed SOI1 waveguides indeed exhibit the largest $\eta$ values.

To extract the $\chi^{(2)}$ values from the measured efficiencies $\eta$, a standard model was applied ${ }^{1}$. As no phase-matching mechanism is present in our waveguides, the model for unphase-matched SHG should be used. In this case, the theory predicts a spectral and longitudinal spatial periodic dependence of $\eta$ (refs 1,21). Namely, $\eta$ is a periodic function of the pump wavelength, with a period of about $1.5 \mathrm{~nm}$ (ref. 21). Numerically solving the nonlinearly coupled equations for pump and second-harmonic waves including dispersion at both harmonics ${ }^{22}$ shows that the $10 \mathrm{~nm}$ pump laser bandwidth completely masks the fast spectral oscillations: the single-peaked dependence of the second-harmonic power on the pump wavelength shown in Fig. 5d can thus be reproduced. On the other hand, it turns out that the second-harmonic signal is still a periodic function of the propagation distance: the second-harmonic signal power periodically oscillates between zero and its maximum value each coherence length (which is $\sim 5.8 \mu \mathrm{m}$ for the SOI1 $10.7-\mu \mathrm{m}$-wide waveguide). Therefore only a lower bound for $\chi^{(2)}$ can be extracted from $\eta$ by considering that the sample length is exactly equal to an odd multiple of the coherence length. In this case, and in the undepleted-pump approximation ${ }^{1}$,

$$
\eta=\frac{8 \pi^{2}\left[\chi^{(2)}\right]^{2} P_{\omega}}{\epsilon_{0} c n_{\omega}^{2} n_{2 \omega} A \lambda_{\omega}^{2}} \frac{1}{\Delta k^{2}}
$$

where $\epsilon_{0}$ is the free-space electric permittivity, $\Delta k=k_{2 \omega}-2 k_{\omega}=$ $2 \pi\left(n_{2 \omega} / \lambda_{2 \omega}-2 n_{\omega} / \lambda_{\omega}\right)$ is the phase mismatch between the pump and the second-harmonic signal, $n_{\omega}$ and $n_{2 \omega}$ are the effective refractive indices at the pump and second-harmonic wavelengths, $\lambda_{\omega}$ and $\lambda_{2 \omega}$ are the pump and second-harmonic wavelengths and $A$ is the modal cross-section, which for simplicity is taken to be equal to half of the waveguide cross-section.

Table 1 provides the calculated $\chi^{(2)}$ values, which demonstrate that a $\chi^{(2)}$ of several tens of picometres per volt can be achieved by cladding the $\mathrm{Si}$ waveguides with a $\mathrm{SiN}_{x}$ overlayer. Although a considerable $\chi^{(2)}$ generation at the interface cannot be completely ruled out, we believe that the strong measured SHG is caused by the inhomogeneous strain in the bulk Si waveguides. The SOI1 $10.7-\mu \mathrm{m}$-wide waveguide exhibits the largest value of $\chi^{(2)}$. Note that this waveguide is the widest in the SOI 1 set, and its optical modes are the most confined within the waveguide core. This observation confirms that the $\chi^{(2)}$ has a bulk origin with a minor contribution from surface effects. The observed dependence of $\chi^{(2)}$ on the stressing layer and, in particular, on the strain inhomogeneity also points to a bulk origin of $\chi^{(2)}$. Moreover, there exists a qualitative agreement between the strain distribution of our waveguides and that of the strained Si model structures. In particular, the SOI1 waveguide presents a sign inversion of the strain as in the model structure of Fig. 1, where both compressive and tensile strain were applied. Instead, the waveguides SOI2 and SOI3 are qualitatively similar to the purely tensile model structures (Supplementary Information).

As the second-order nonlinearity is related to the local lattice deformation (that is the local strain) and the Raman measurements show that the strain is very inhomogeneous in the waveguide, $\chi^{(2)}$ is expected to have a distribution of values across the waveguide. On the other hand, the nonlinear transmission measurements are averaging in a non-simple way the $\chi^{(2)}$ over the waveguide cross-section, because the second-harmonic efficiency depends on the overlap integral between the pump and the second-harmonic 
a

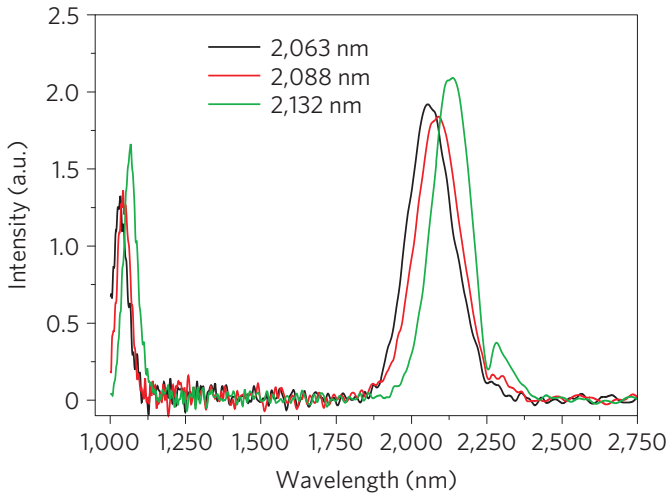

c

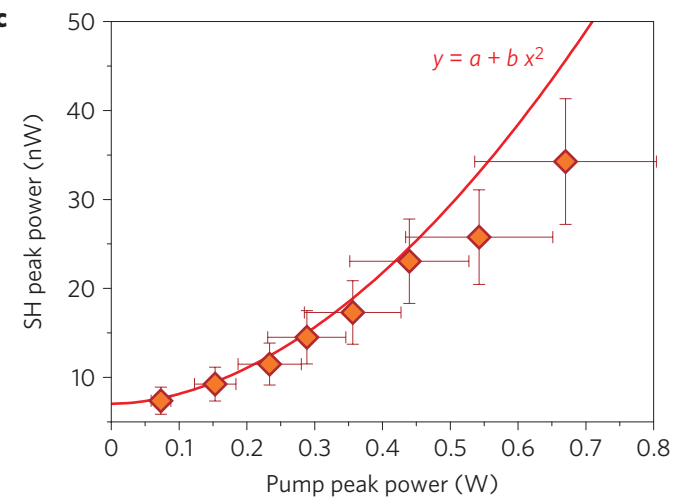

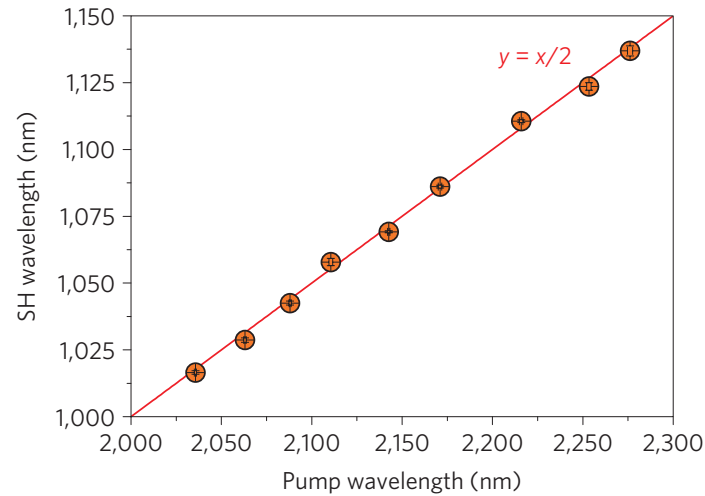

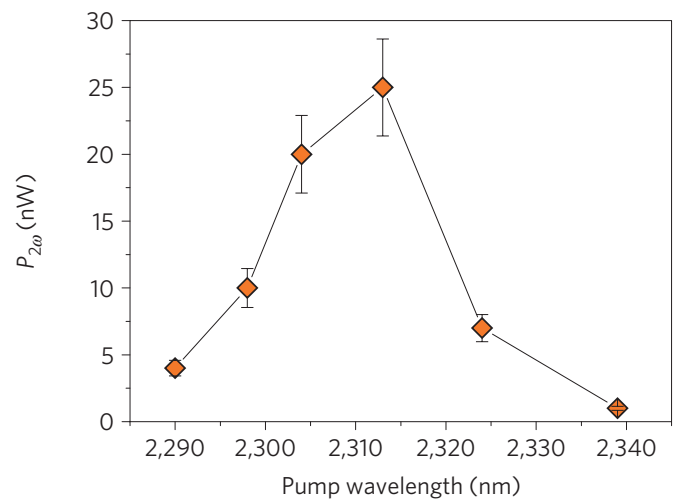

Figure 5 | Summary of the SHG measurements. a, SOI1 10.7- $\mu \mathrm{m}$-wide waveguide transmission spectra with a fs pump and peak power of $30 \mathrm{~kW}$. b, Second-harmonic peak tunability curve with fs-pumping peak power $2 \mathrm{~kW}$ on SOl1 10.7- $\mu \mathrm{m}$-wide waveguide. The data are measured values and the line shows the expected relationship between $\lambda_{2 \omega}$ and $\lambda_{\omega}$. c, Power dependence of the second-harmonic signal for the SOI1 2.3- $\mu \mathrm{m}$-wide waveguide and $\lambda_{\omega}=2,313 \mathrm{~nm}$. The ns pump source was used. The line is a power-law fit to the data points, where the fitted power exponent is $1.9 \pm 0.4$. The deviation of points from the fitting line at high powers is due to the onset of nonlinear absorption, which depletes the pump. $\mathbf{d}$, Dependence of the second-harmonic peak power $P_{2 \omega}$ on the pump wavelength $\lambda_{\omega}$ for the SOl1 2.3- $\mu \mathrm{m}$-wide waveguide and a pump peak power of $0.7 \pm 0.1 \mathrm{~W}$ of the ns source. The error bars in b-d are the maximum propagation errors from repeated measurements.

Table 1 | Summary of the relevant parameters of the investigated waveguides.

\begin{tabular}{|c|c|c|c|c|c|c|}
\hline Waveguide & $w_{0}(\mu \mathrm{m})$ & $h_{0}(\mu \mathrm{m})$ & $n_{\omega}$ & $n_{2 \omega}$ & $\eta\left(\mathbf{W ~ W ~}^{-1}\right)$ & $\chi^{(2)}\left(p m V^{-1}\right)$ \\
\hline SOI1 & $\begin{array}{l}2.3 \\
2.5 \\
10.7\end{array}$ & $\begin{array}{l}2.1 \\
2.0 \\
2.0\end{array}$ & $\begin{array}{l}3.371 \\
3.374 \\
3.404\end{array}$ & $\begin{array}{l}3.510 \\
3.510 \\
3.518\end{array}$ & $\begin{array}{l}(3 \pm 2) \times 10^{-8} \\
(5 \pm 2) \times 10^{-8} \\
(5 \pm 3) \times 10^{-8}\end{array}$ & $\begin{array}{l}20 \pm 15 \\
20 \pm 15 \\
40 \pm 30\end{array}$ \\
\hline SOI2 & $\begin{array}{l}2.0 \\
2.5 \\
11.7\end{array}$ & $\begin{array}{l}2.5 \\
2.3 \\
2.5\end{array}$ & $\begin{array}{l}3.371 \\
3.381 \\
3.415\end{array}$ & $\begin{array}{l}3.510 \\
3.512 \\
3.521\end{array}$ & $\begin{array}{l}(1 \pm 0.5) \times 10^{-8} \\
(5 \pm 2) \times 10^{-9} \\
(4 \pm 2) \times 10^{-10}\end{array}$ & $\begin{array}{l}11 \pm 8 \\
8 \pm 5 \\
4 \pm 3\end{array}$ \\
\hline SOI3 & $\begin{array}{l}2.0 \\
2.3 \\
11.7\end{array}$ & $\begin{array}{l}2.2 \\
2.2 \\
2.2\end{array}$ & $\begin{array}{l}3.362 \\
3.375 \\
3.409\end{array}$ & $\begin{array}{l}3.507 \\
3.511 \\
3.519\end{array}$ & $\begin{array}{l}(6 \pm 3) \times 10^{-9} \\
(9 \pm 4) \times 10^{-9} \\
(5 \pm 3) \times 10^{-9}\end{array}$ & $\begin{array}{l}9 \pm 6 \\
10 \pm 7 \\
14 \pm 10\end{array}$ \\
\hline
\end{tabular}

The first column refers to the differently stressed waveguides, where SOI1 refers to a tensile stressing overlayer, SOI2 refers to the compressive stressing overlayer and SOI 3 refers to the stress-compensated overlayer. The second and third columns give the geometrical parameters of the waveguide cross-section (Fig. $2 a$ ). $n_{\omega}$ and $n_{2 \omega}$ are the effective indices for the fundamental modes at the pump $\left(\lambda_{\omega}=2,313 \mathrm{~nm}\right)$ and second-harmonic $\left(\lambda_{2 \omega}=1,156.5 \mathrm{~nm}\right)$ wavelengths. $\eta$ is the measured power-conversion efficiency and $\chi^{(2)}$ the second-order susceptibility calculated with equation (1) using the $\eta$ values. All measurements refer to a ns pump peak power of $0.7 \pm 0.1 \mathrm{~W}$.

signal as well as on the $\chi^{(2)}$ distribution. Therefore, we can point out that the observed experimental trend is related to the inhomogeneity and magnitude of the strain and, indeed, simulations show that experiments can be well accounted by the model. Thus, both theory and experiments clearly demonstrate the possibility to induce second-order nonlinear response from bulk Si by using strain.

It is worth noting the fact that recent reports on surfacegenerated SHG in silicon nitride waveguides ${ }^{23}$ or in Si photonic crystal nanocavities ${ }^{12}$ provided $\chi^{(2)}$ values that are significantly smaller than the bulk induced $\chi^{(2)}$ observed at present. Noticeably, the $\chi^{(2)}$ values reported here are similar to the one found in refs 13 and 14 . We should also note that the values of $\chi^{(2)}$ which were measured by surface reflection spectroscopy in $\mathrm{Si}$ also showed that significant strain-induced enhancement of secondorder nonlinearities with respect to surface-originated $\chi^{(2)}$ values are possible $e^{9,10}$. Strained Si waveguides can achieve a nonlinearity competitive in magnitude with the widely used $\mathrm{LiNbO}_{3}$. Such values are large enough to permit the development of practical optical integrated devices based on bulk second-order nonlinearities of Si. 
In conclusion, we have shown theoretically and demonstrated experimentally a bulk second-order dipolar nonlinear optical susceptibility in $\mathrm{Si}$ waveguides, which were strained by $\mathrm{SiN}_{x}$ overlayers. We believe that such a second-order susceptibility is caused by the inhomogeneous strain in the Si leading to a strong dependence of $\chi^{(2)}$ on the extent and the inhomogeneity of the induced strain field. In particular, the best situation is observed when the stress is biaxial and the resulting strain has an inversion in sign across the waveguide. In this case, second-harmonic conversion efficiencies of the order of $10^{-8}$ under ns pump were observed. These observations provide a lower bound to $\chi^{(2)}$ of $40 \mathrm{pm} \mathrm{V}^{-1}$, which is three orders of magnitude higher than the reported $\sim 10^{-2} \mathrm{pm} \mathrm{V}^{-1}$ value due to surface effects ${ }^{12,23}$. It is worth noticing that no phase-matching mechanism was employed, thus leaving room for significantly boosting the output SHG power by many orders of magnitude by introducing an appropriate periodic poling by strain alternation ${ }^{24}$, or by multimode phase-matching techniques ${ }^{23}$. The combination of strained $\mathrm{Si}$ with suitable photonic design can lead to nonlinear Si devices that may compete with devices based on conventional nonlinear materials but with the immense advantages connected with Si photonic integrated circuits, which are potentially compatible with mass manufacturing.

\section{Methods}

$\boldsymbol{A} \boldsymbol{b}$-initio simulations. To simulate strained bulk Si we used the supercell technique with a unit cell of 16 atoms. Si symmetry is broken by moving some of the Si atoms, that is by modifying the Si bond lengths and angles (up to 5\% with respect to unstrained bulk). With this method, we introduced tensile-tensile or compressive-tensile strain. The electronic structure of the systems in their ground state was computed with density functional theory in the local density approximation, by using norm-conserving pseudopotentials and a plane-wave basis set with the code ABINIT (refs 25,26$)$. Then, $\chi^{(2)}\left(\lambda_{\omega}\right)$ was computed for SHG spectroscopy in time-dependent density-functional theory with the 2 light code based on the formalism developed in refs 16,17 . The spectra were calculated in the independent particle approximation using for the quasiparticle energies a scissor operator of $0.6 \mathrm{eV}$ (ref. 27). Convergence was achieved with 2,166 off-symmetry shifted $k$ points in the full Brillouin zone and 20 unoccupied states. To compute the $\mathrm{Si} / \mathrm{SiO}_{2}$ interface, the $\beta$-cristobalite structure for $\mathrm{SiO}_{2}$ was used. The system was not relaxed, keeping Si bulk properties. The $\chi^{(2)}$ signal only depends on the interface, as by construction no strained bulk contribution to $\chi^{(2)}$ was considered.

See Supplementary Information for all the details of the theoretical calculations.

Device fabrication. Strained Si waveguides were realized starting with (100)-oriented prime SOI wafers (Si-Mat) with a $2.2-\mu \mathrm{m}$-thick Si device layer and $2-\mu \mathrm{m}$-thick buried thermal oxide layer. Silicon nitride overlayers were deposited on SOI wafers by using different deposition techniques. The resulting stress induced by the silicon nitride overlayer in the SOI wafer was calculated by monitoring the change in the bending radius of unpatterned wafers through both mechanical and optical profilometry. In particular, a 150 -nm-thick $\mathrm{Si}_{3} \mathrm{~N}_{4}$ layer, deposited at $780^{\circ} \mathrm{C}$ using a low-pressure chemical vapour deposition technique, results in a 1.2 GPa tensile stress (wafer SOI1). A parallel-plate plasma-enhanced chemical vapour deposition technique was used to deposit a $500-\mathrm{nm}$-thick $\mathrm{SiN}_{x}$ layer at $300^{\circ} \mathrm{C}$ (plasma frequency $308 \mathrm{kHz}$ ) with a resulting $-500 \mathrm{MPa}$ compressive stress (wafer SOI2). A low-stress 500-nm-thick $\mathrm{SiN}_{x}$ layer ( $-60 \mathrm{MPa}$, compressive) was deposited by two-frequency plasma-enhanced chemical vapour deposition (wafer SOI3). In this case, during the deposition we used an alternation between the high and low plasma frequencies ( $13.56 \mathrm{MHz}$ for $50 \mathrm{~s}$ and $308 \mathrm{kHz}$ for $10 \mathrm{~s}$ ), thus compensating the accumulated stress after each $1 \mathrm{~min}$ deposition cycle. Strained $\mathrm{Si}$ waveguides were patterned lithographically and dry-etched anisotropically down to the BOX layer in a plasma etcher using $\mathrm{CHF}_{3}-\mathrm{SF}_{6}$ gas chemistry. A control wafer (named SOI0) without the stressing overlayer was used to fabricate reference waveguides. The residual stress in the SOI substrates due to the Si device layer and the BOX was also evaluated by measuring the wafer curvatures after a step by step removal of the device layer and BOX. These measurements show that the stress of the Si device layer is negligible, whereas the oxide layer (BOX) induces a stress of $-318 \mathrm{MPa}$.

Micro-Raman experiments. The strain maps were measured by scanning the waveguide cross-section on the [110] facet with a confocal Raman microscope (Witec SNOM alpha $300 \mathrm{~S}$ ) in backscattering configuration. A $150 \mathrm{~mW}$ Ar laser at $514.5 \mathrm{~nm}$ and a spectrometer with a grating of 1,800 grooves $\mathrm{mm}^{-1}$ coupled to a CCD (charge-coupled device; Andor DU401) were used. The Raman image is obtained by acquiring a complete Raman spectrum for each point, with a pixel step of $0.25 \mu \mathrm{m} \times 0.25 \mu \mathrm{m}$, and by fitting the spectrum with a Lorentz function to estimate the Raman peak wavenumber with an error of about $0.05 \mathrm{~cm}^{-1}$. The strain-induced shift of the Si Raman peak was determined with reference to the relaxed Si Raman peak measured in the substrate. Typical shifts in the range (0.5, $-1 \mathrm{~cm}^{-1}$ ) were observed. The distribution of strain components was constructed by solving point by point the equations relating the strain-tensor components $\left(\varepsilon_{X X}, \varepsilon_{Y Y}, \ldots\right)$ to the Raman peak shift within the uniaxial-stress model

$$
\begin{gathered}
\varepsilon_{X X}=\left(\frac{S_{11}+S_{12}}{2}-\frac{S_{44}}{4}\right) \sigma_{Y Y} \\
\varepsilon_{Y Y}=\left(\frac{S_{11}+S_{12}}{2}+\frac{S_{44}}{4}\right) \sigma_{Y Y} \\
\varepsilon_{Z Z}=S_{12} \sigma_{Y Y} \\
\varepsilon_{X Z}=\varepsilon_{Y Z}=\varepsilon_{X Y}=0
\end{gathered}
$$

where $S_{11}=7.68 \times 10^{-12} \mathrm{~Pa}^{-1}, S_{12}=-2.14 \times 10^{-12} \mathrm{~Pa}^{-1}, S_{44}=12.7 \times 10^{-12} \mathrm{~Pa}^{-1}$ are the elastic compliance parameters of $S i$ and $X, Y$ and $Z$ correspond to the (110), (-110) and (001) crystallographic directions. The stress-tensor component $\sigma_{Y Y}$ is related to the Raman peak shift according to $\Delta \omega \approx-\mathcal{C} \omega_{0} \sigma_{Y Y}$, where $\omega_{0}$ is the stress-free frequency of the Si Raman peak and $\mathcal{C} \sim 3.9 \times 10^{-12} \mathrm{~Pa}^{-1}$ was deduced from measurements ${ }^{28}$.

Nonlinear-transmission experiments. Two pump lasers were used: a 4 ns optical parametric oscillator (Newport Spectra-Physics MOPO-PO 710) pumped by a Nd:yttrium aluminium garnet laser operating at $10 \mathrm{~Hz}$ repetition rate and a $\sim 100 \mathrm{fs}$ tunable laser (a travelling-wave optical parametric amplifier TOPAS-C from Light Conversion pumped by a Newport Spectra-Physics SPITFIRE regenerative amplifier, $35 \mathrm{fs}$ operating at $1 \mathrm{kHz}$ repetition rate). For the ns experiment the second-harmonic beam was analysed by a fibre-coupled monochromator equipped with a liquid-nitrogen-cooled InGaAs photomultiplier (Hamamatsu) interfaced to a photon-counting multichannel scaler (model SR430 from Stanford Research Systems) triggered by the pump laser pulses. The InGaAs photomultiplier is sensitive in the $800-1,700 \mathrm{~nm}$ range. For the fs experiment the transmitted beam was imaged through a two-blade pinhole into a Fourier-transform infrared spectrometer model Vertex70v (Bruker) equipped with a 77-K-cooled InSb detector interfaced to a lock-in amplifier. The waveguides were mounted on nanopositioners and the pump signal was butt-coupled by using reflective gold-coated objectives. Note that the power value reported in the manuscript refers to the power coupled into the waveguide estimated on the basis of the measured coupling losses. The errors in the second-harmonic conversion efficiency come from the propagation of the errors of repeated nonlinear transmission measurements and represent the maximum errors, that is, the half-width of the measurement distribution.

\section{Received 22 June 2011; accepted 14 November 2011; published online 4 December 2011}

\section{References}

1. Sutherland, R. L. Handbook of Nonlinear Optics 2nd edn (CRC Press, 2003).

2. Pavesi, L. \& Lockwood, D. Silicon Photonics I (Topics in Applied Physics Vol. 94, Springer, 2004).

3. Lockwood, D. \& Pavesi, L. Silicon Photonics II (Topics in Applied Physics Vol. 119, Springer, 2011).

4. Lin, Q., Painter, O. J. \& Agrawal, G. P. Nonlinear optical phenomena in silicon waveguides: Modeling and applications. Opt. Express 25, 16604-16644 (2007).

5. Leuthold, J., Koos, C. \& Freude, W. Nonlinear silicon photonics. Nature Photon. 4, 535-544 (2010).

6. Guidotti, D. \& Driscoll, T. A. Second-harmonic generation in centro-symmetric semiconductors. Nuovo Cimento. 8D, 385-416 (1986).

7. Huang, J. Y. Probing inhomogeneous lattice deformation at interface of $\mathrm{Si}(111) / \mathrm{SiO}_{2}$ by optical second-harmonic reflection and Raman spectroscopy. Jpn. J. Appl. Phys. 33, 3878-3886 (1994).

8. Govorkov, S. V. et al. Inhomogeneous deformation of silicon surface layers probed by second-harmonic generation in reflection. J. Opt. Soc. Am. B 6, $1117-1124$ (1989).

9. Zhao, Ji-H. et al. Enhancement of second-harmonic generation from silicon stripes under external cylindrical strain. Opt. Lett. 34, 3340-3342 (2009).

10. Schriever, C., Bohley, C. \& Wehrspohn, R. B. Strain dependence of second-harmonic generation in silicon. Opt. Lett. 35, 273-275 (2010).

11. Mitchell, S. A., Mehendale, M., Villeneuve, D. M. \& Boukherroub, R. Second harmonic generation spectroscopy of chemically modified $\mathrm{Si}(111)$ surfaces. Surf. Sci. 488, 367-378 (2001).

12. Galli, M. et al. Low-power continuous-wave generation of visible harmonics in silicon photonic crystal nanocavities. Opt. Express 18, 26613-26624 (2010).

13. Jacobsen, R. S. et al. Strained silicon as a new electro-optic material. Nature 441, 199-202 (2006). 
14. Chmielak, B. et al. Pockels effect based fully integrated, strained silicon electro-optic modulator. Opt. Express 19, 17212-17219 (2011).

15. Hon, N. K., Tsia, K. K., Solli, D. R., Jalali, B. \& Khurgin, J. B. Stress-induced $\chi^{(2)}$ in silicon-comparison between theoretical and experimental values. Proc. 6th IEEE Int. Conf. Group IV Photonics 232-234 (2009).

16. Luppi, E., Hübener, H. \& Véniard, V. Ab-initio second-order nonlinear optics in solids. J. Chem. Phys. 132, 241104 (2010).

17. Luppi, E., Hübener, H. \& Véniard, V. Ab-initio second-order nonlinear optics in solids: Second-harmonic generation spectroscopy from time-dependent density-functional theory. Phys. Rev. B 82, 235201 (2010).

18. Hübener, H., Luppi, E. \& Véniard, V. Ab initio calculation of many-body effects on the second-harmonic generation spectra of hexagonal SiC polytypes. Phys. Rev. B 83, 115205 (2011).

19. Mejia, J. E. et al. Surface second-harmonic generation from $\operatorname{Si}(111)(1 \times 1) \mathrm{H}$ : Theory versus experiment. Phys. Rev. B 66, 195329 (2002).

20. De Wolf, I., Maes, H. E. \& Jones, S. K. Stress measurements in silicon devices through Raman spectroscopy: Bridging the gap between theory and experiment. J. Appl. Phys. 79, 7148-7156 (1996).

21. Fejer, M. M., Magel, G. A., Jundt, D. H. \& Byer, R. L. Quasi-phase matched second harmonic generation-tuning and tolerances. IEEE J. Quantum Electron. 28, 2631-2654 (1992).

22. Liu, X., Qian, L. J. \& Wise, F. W. Generation of optical spatiotemporal solitons. Phys. Rev. Lett. 82, 4631-4634 (1999).

23. Levy, J. S., Foster, M. A., Gaeta, A. L. \& Lipson, M. Harmonic generation in silicon nitride ring resonators. Opt. Express 19, 11415-11421 (2011).

24. Hon, N. K., Tsia, K. K., Solli, D. R \& Jalali, B. Periodically poled silicon. Appl. Phys. Lett. 94, 091116 (2009).

25. Gonze, X. et al. ABINIT: First-principles approach to material and nanosystem properties. Comput. Phys. Commun. 180, 2582-2615 (2009).
26. Gonze, X. et al. A brief introduction to the ABINIT software package. Z. Kristallogr. 220, 558-562 (2005).

27. Godby, R. W., Schluter, M. \& Sham, L. J. Self-energy operators and exchange-correlation potentials in semiconductors. Phys. Rev. B 37, 10159-10175 (1988).

28. Bianco, F. et al. To be published.

\section{Acknowledgements}

We acknowledge discussions and experimental help by P. Bettotti, A. Pitanti, B. Dierre, F. Enrichi, K. Fedus and A. Yeremian. This work was supported by the FU-PAT (Provincia Autonoma di Trento) project NAOMI, by a grant from Fondazione Cariplo no 2009-2730 and by Fondazione Cassa di Risparmio di Modena through the project 'Progettazione di materiali nanostrutturati semiconduttori per la fotonica, l'energia rinnovabile e l'ambiente'. We also acknowledge the supercomputing facility CINECA for granted central processing unit time.

\section{Author contributions}

M.C. and L.P. conceived the experiments. E.B., F.B. and M.C. made the nonlinear optical measurements. M.G. and G.P. fabricated the waveguides. E.D., E.L., V.V. and S.O. carried out the $a b$ initio simulations. D.M. and S.W. did the nonlinear propagation modelling. R.P. and F.B. made the micro-Raman measurements. L.P. wrote the manuscript in collaboration with all the authors.

\section{Additional information}

The authors declare no competing financial interests. Supplementary information accompanies this paper on www.nature.com/naturematerials. Reprints and permissions information is available online at http://www.nature.com/reprints. Correspondence and requests for materials should be addressed to L.P. 